Sara Hawtrey Jones, Department of Mathematics, University of Southern

Colorado, Pueblo, CO 81001-4901, e-mail: sara@meteor.uscolo.edu

\title{
Applications of the Baire Category Theorem
}

\section{Contents}

1 Introduction 363

2 Spaces of Functions $\quad 365$

3 Analytic and $C^{\infty}$-functions $\quad 367$

4 Typical Intersection Patterns $\quad 368$

5 Iteration of Continuous Functions $\quad 369$

6 Kolmogorov's Theorem 380

$\begin{array}{lll}7 & \text { Pseudo-arcs and subsets of the plane } & 382\end{array}$

\section{Introduction}

In his doctoral thesis in 1899, René Baire writes, "The continuum constitutes a set of the second category." This is one of the the statements for which Baire is best known. However, the development of this idea barely takes a page in Baire's 122 page thesis. The goal of Baire's thesis was to characterize those functions of two variables which may not be continuous but are continuous in each variable separately. By the end of his first 27 pages, Baire had shown that these functions were pointwise discontinuous on each perfect set. (A function is pointwise discontinuous with respect to a closed set if the set of points of continuity of the function restricted to this set is dense with respect to the set.) The term 'pointwise discontinuity' may be misleading because such functions are actually continuous on a large subset of their domain. Baire showed that

Key Words: Baire Category Theorem, typical, typical continuous functions, $\omega$-limit sets, Level sets, Intersection Patterns

Mathematical Reviews subject classification: 26A18 and 26A15

Received by the editors October 19, 1997 
these functions can be represented as pointwise limits of convergent sequences of continuous functions. Such functions later became known as functions of Baire class 1 , or simply $B_{1}$ functions. Baire showed that a function can be obtained as a convergent sequence of continuous functions if and only if it is pointwise discontinuous on each perfect subset of its domain.

Near the end of Chapter two of his thesis, Baire introduced the concepts of first and second category. To define first category, Baire relied on Cantor's definition of dense (a set is dense if its closure is the whole space [28, vol. 15, p.2] and on P. du Bois-Reymond's definition of nowhere dense (a set is nowhere dense if the complement of its closure is dense $[\mathrm{DuB}][\mathrm{Ku}$, Vol I, p. 66]. A nowhere dense set $A$, has the property that for each open subset of the space, there is an open ball contained in this set which does not intersect $A$ ). A set is first category if it can be written as a countable union of nowhere dense sets and is second category set if it is not first category [Bai, p. 65]. (Following Denjoy, we call sets which are the complements of first category sets, residual [Den3]. If each member of a residual set has a certain property, we will call that property typical.)

Within a paragraph of introducing the concept of category, Baire concluded that the continuum is not of the first category and made the point that first category subsets of the real line, $\mathbf{R}$, are small. Baire then showed that the set of points of discontinuity of a $B_{1}$ function is first category. Baire extended these results by showing that the classes of semicontinuous functions, functions of bounded variation, and derived functions are all contained in the class $B_{1}$. Thus, the above classes of functions all have small sets of points of discontinuity.

It took twenty more years for the generalization of the Baire Category theorem to be applied to other complete metric spaces.

Theorem 1 (Baire). Let $(X, \rho)$ be a complete metric space, and $S$ be a countable union of nowhere dense sets in $X$. Then the complement of $S$ is dense in $X$.

The definitions for first category, second category, residual, and typical all apply in any complete metric space. Baire's theorem guarantees that the space cannot be written as a countable union of nowhere dense sets. In fact, the complement of a first category set is dense in the space and large in the sense of category, this set is residual. Sometimes, the Baire Category Theorem will provide a proof for the existence of functions and sets with certain properties more easily than providing a construction of a function or set with the property. 


\section{Spaces of Functions}

First consider the space of all continuous functions, $f:[0,1] \rightarrow[0,1]$ denoted by $C(I, I)$ where $I=[0,1]$ or simply $C$ if there is no confusion. Distance is given by the sup metric:

$$
|f-g|=\sup _{x \in I}|f(x)-g(x)|
$$

With this metric, $C$ is a complete metric space, so the Baire Category Theorem holds. (Fréchet showed that this space was a complete space in 1904, but according to Fréchet, this distance between functions was previously considered by Weierstrass [39].) Around 1930, numerous authors applied existence arguments via the Baire Category Theorem to prove the existence of functions of real variables exhibiting certain singularities [58, Vol. I, p. 420]. For example, Mazurkiewicz and Banach used the category method to show the existence of a continuous function without a finite derivative at any point [64] and $[5$, p. 327].

Theorem 2 (Banach). The typical continuous function has no finite derivative at any point.

The proof of this result involves a sequence of dense open sets which contain functions with steep slopes. These sets are complements of the sets

$$
E_{n}=\{f \in C: \exists x \in[0,1-1 / n] \ni|f(x+h)-f(x)| \leq n h, \forall h \in(0,1-x)\} .
$$

After showing that the sets $E_{n}$ are closed, they are shown to be nowhere dense by showing that their complements are dense. Let $\epsilon>0$. For each $f \in C$ find a piecewise linear function $g$ so that $|f-g|<\epsilon / 2$. For any such $g$, find a function $h$ so that $|h-g|<\epsilon$ and $h \notin E_{n}$ as follows. Let $M$ be the maximum absolute value of the slopes of the line segments which constitute $g$. Choose $m$ so that $m \epsilon>n+M$. Define

$$
\phi(x)=\min (x-[x],[x]+1-x)
$$

The function $\phi(x)$ gives the distance from $x$ to the nearest integer. Set $h(x)=$ $g(x)+\epsilon \phi(m x)$. Then for each point of $[0,1), h$ has a one sided derivative on the right greater than $m \epsilon+M>n$, so $h \in C-E_{n}$. Since $|g-h|=\epsilon / 2$. Thus the complement of $E_{n}$ is dense. See for example [75, p.45], [58, Vol. I, p. 240], [88, p.299] [21, p.463].

Weierstrass had previously given an example of a nowhere differentiable continuous function as a sum of a uniformly convergent series of sawtooth functions. In this way, the Baire Category Theorem can sometimes be used to 
construct the functions for which it is used to show existence. But, as Oxtoby points out, "if the proof of nowhere denseness is indirect or long and involved, it may be difficult to obtain an explicit example in this way [75, p.46]."

Use of the Baire Category theorem helps mathematicians prove the existence of functions that were very difficult to construct and even more difficult to visualize. For example, during the 1800's mathematicians could not determine if differentiable nowhere monotonic functions actually exist. In order for a differentiable function in $C$ to be nowhere monotonic it must be true that both the sets $\left\{x: f^{\prime}(x)>0\right\}$ and $\left\{x: f^{\prime}(x)<0\right\}$ are dense in $[0,1]$. Dini believed that such functions probably existed while du Bois-Reymond believed that nowhere monotonic functions could not be differentiable. In 1887, Köpcke provided a complicated construction of a differentiable nowhere monotonic function. This construction was followed by many others given by Denjoy, Pereno, Hobson, and Köpcke himself [31], [46]. Some of the shorter constructions were just ten pages long. Then in 1976, C. Weil used of the Baire Category Theorem to prove the existence of such functions in 2 pages [90]. The statement of this theorem gives an idea as to how it was proved. See also, [19], [21, p. 465].

Theorem 3 (Weil). Let $\Delta_{0}$ be the set of differentiable functions $F$ on the interval $[0,1]$ such that $F(0)=0$ and $F^{\prime}$ is bounded and defined for all $x \in[0,1]$ and $F(x)=0$ for a dense subset of $[0,1]$. For $F, G \in \Delta_{0}$ let

$$
\rho(F, G)=\sup _{x \in[0,1]}\left|F^{\prime}(x)-G^{\prime}(x)\right| .
$$

then $\left(\Delta_{0}, \rho\right)$ is a complete metric space in which the typical member is a differentiable nowhere monotonic function [90].

In some cases the Baire Category Theorem was the first method used to show the existence of functions with certain properties. For instance, in 1931, Oxtoby used the category method to show the existence of a transitive homeomorphism $T$ of the unit square. If $f^{1}\left(x_{0}\right)=f\left(x_{0}\right)$, and $f^{n}\left(x_{0}\right)=$ $f\left(f^{n-1}\left(x_{0}\right)\right)$, then a function $f$ is transitive, if for each pair of open sets $U$ and $V$ in $I$, there is a positive integer $k$ such that $f^{k}(U) \cap V \neq \emptyset$. Some spaces admit transitive homeomorphisms and others do not. For example, no automorphism of the unit interval is transitive, but multiplication by $e^{2 \pi i \alpha}$, with $\alpha$ irrational, defines a transitive rotation of the unit circle in the complex plane. An explicit transformation of the complex plane was given by Besicovich [7], but it is not easy to exhibit one for the closed unit square, let alone one that preserves area and leaves boundary points fixed. The existence of such objects was first exhibited by Oxtoby using the category method [76] and [75, p.70]. 


\section{Analytic and $C^{\infty}$-functions}

Baire's theorem illustrates the difference between analytic functions and $C^{\infty_{-}}$ functions. To be analytic at $x_{0}$, the function must have a Taylor series with a positive radius of convergence at $x_{0}$. To be a $C^{\infty}$-function, the $n^{\text {th }}$ derivative must be continuous for every $n$. We can show using the Baire Category Theorem that most $C^{\infty}$-functions are not analytic at any point. This construction was outlined by Morgenstern in his paper Unendlich oft differenzierbare nichtanaytische [73]. (An English version of the proof can be found in [52].)

Theorem 4 (Morgenstern). Typical $C^{\infty}$-functions are not analytic at any point [73].

Great mathematicians like Lagrange, Babbage, Herschel and Peacock believed that every function could be represented by a power series. This assumption was held to be true until as late as 1816. It implies that the value of a function and all of it's derivatives at a certain point completely determine the function for every value of $x$. It was not until Fourier constructed a power series that converged on only the interval $(0,1)$ that this assumption was called into question. This forced a critical reevaluation of what was meant by a function, an infinite series, and a derivative [18, p.57]. In 1821, Cauchy exhibited his famous counterexample to Lagrange's assertion that distinct functions have distinct power series:

$$
f(x)=e^{-\frac{1}{x^{2}}} f(0)=0 .
$$

This function has the same power series at $x=0$ as the constant function $f(x)=0$ [29].

Similar analysis can be done if we consider Fourier series rather than Taylor series. In 1876, P. du Bois-Raymond gave an example of a continuous function defined on $[-\pi, \pi]$ with a Fourier series that diverges at a point. The existence of such a function is easy to prove by using the uniform boundedness principle whose proof relies on the Baire Category Theorem. In fact, the use of a category argument in the proof shows that the typical continuous function has dense $G_{\delta}$ set of points of nonconvergence [21, P. 684]. However, in each case this set has measure zero. (To complete the picture, in 1926, Kolmogorov produced an example of an integrable function whose Fourier series diverges almost everywhere in $[-\pi, \pi]$, but it was still unknown if a continuous function could have a Fourier series which diverged on a set of positive measure. Lusin conjectured that it could not. In 1966, Carleson proved that "Lusin's conjecture" is true. Namely, the Fourier series for every continuous function on $[-\pi, \pi]$ converges almost everywhere $[88$, p.362] or $[21$, p.685]. (Carleson actually showed that the Fourier series for every $L^{2}$ function converges almost everywhere.)) 


\section{Typical Intersection Patterns}

This section involves intersection patterns of continuous functions with various sets. Let us turn to the intersection patterns that a typical continuous function exhibits. For $f \in C$, let $M_{f}=\max \left\{f(x): x \in I_{0}\right\}$ and $m_{f}=\min \{f(x)$ : $\left.x \in I_{0}\right\}$. Theorem 5 provides the typical intersection pattern for a continuous function with the family of horizontal lines [19, chapter 13] or [24].

Theorem 5 (Bruckner and Garg). To the typical continuous function in $C$ there corresponds a countable dense subset, $S_{f}$, of $\left(m_{f}, M_{f}\right)$ such that the set $E_{\alpha}=\{x: f(x)=\alpha\}$ is

(i) a single point when $\alpha=m_{f}$ or $\alpha=M_{f}$.

(ii) a nowhere dense perfect set when $\alpha \notin S_{f} \cup\left\{m_{f}, M_{f}\right\}$.

(iii) of the form $P_{\alpha} \cup\left\{x_{\alpha}\right\}$ where $P_{\alpha}$ is a nonempty, nowhere dense, perfect set and $x_{\alpha}$ is isolated in $E_{\alpha}$ when $\alpha \in S_{f}$.

The typical intersection pattern for the family of all lines, given below requires additional notation. For $f \in C$ and $\gamma \in \mathbf{R}$, define a function $f_{\gamma}$ by

$$
f_{\gamma}(x)=f(x)-\gamma x .
$$

Theorem 6 (Bruckner and Garg). To the typical continuous function in $C$ there corresponds a countable dense subset, $\Gamma_{f}$, of $\mathbf{R}$ such that

i) if $\gamma \in \mathbf{R}-\Gamma_{\mathrm{f}}$, the function $f_{\gamma}$ has the same intersection pattern as described in Theorem 5, and

ii) if $\gamma \in \Gamma_{f}$, the function $f_{\gamma}$ meets all conditions of Theorem 5 except that there is a single exceptional level set that contains two isolated points instead of one.

Typical continuous functions are very much alike with respect to the intersection patterns of their graphs with lines. One such function differs from another primarily in which heights or directions are exceptional. Theorem 6 has been extended by Wójtiwicz [91]. He replaces the family of lines by the graphs of certain other two parameter families of functions.

Thus, the graph of a typical continuous function $f$ intersects many lines in uncountable sets - the intersections are large in terms of cardinality. This is expected in terms of the results from the first section because the typical continuous function is nowhere differentiable (therefore, nowhere monotonic, nowhere of bounded variation and nowhere convex or concave). However the 
intersections are small with respect to porosity. This will help explain why the behavior of a typical $f \in C$ is pathological with respect to differentiation.

The porosity $p(x)$, of a subset $E$ of $I$ at a point $x \in E$ is defined as follows. For $h>0$ let $l(x, h)$ be the length of the longest interval contained in $(I-E) \cap(x-h, x+h)$. The quantity

$$
p(x)=\limsup _{h \rightarrow 0} \frac{l(x, r)}{h}
$$

is called the porosity of $E$ at $x$. When $p(x)>0$ for all $x \in E, E$ is said to be porous. If $E$ is porous then $\mathrm{E}$ is nowhere dense. Also, $\lambda(E)=0$ where $\lambda$ represents Lebegue measure, (this follows from Lebesgue's Density Theorem). A set $E$ for which $p(x)=1$ for all $x \in E$ is called strongly porous.

Theorem 7 (Bruckner and Haussermann). For the typical $f \in C$, if $g$ is differentiable on I, then $E_{g}=\{x: f(x)=g(x)\}$ is a strongly porous set [25].

Theorem 7 clarifies why the typical $f \in C$ is nowhere differentiable with respect to so many notions of generalized differentiability. For example, recall that $f$ is approximately differentiable at $x$, if and only if there is a set $E$ having $x$ as a point of density such that $f \mid E$ is differentiable at $x[21$, p. 316]. Any set $E$ having $x$ as a point of density is nonporous at $x$. From $E$, one can select a countable closed set $A=\left\{a_{n}\right\}$ containing $x$ as an accumulation point, with $A$ nonporous at $x$. The function $f \mid A$ can be extended to a differentiable function, $g$, on all of $I$. Thus $\{\alpha: f(\alpha)=g(\alpha)\}$ is nonporous at $x$. It now follows from Theorem 7 that the typical $f \in C$ is nowhere approximately differentiable. More about the intersection patterns of continuous functions can be found in [26].

\section{$5 \quad$ Iteration of Continuous Functions}

In this section, we will see what the Baire Category Theorem has to say about the behavior of continuous functions with respect to iteration. More specifically, we want to contrast the behavior of a typical continuous function with the behavior of a "nice" function that is usually studied in dynamical systems. We will discuss the dynamics of one dimensional maps, that is, of continuous maps of $[0,1]$ onto itself. The study of iteration of a continuous map arose from the study of populations or processes that evolve discretely over time. Problems involving how a population or a process develop over time can be modeled by differential equations if the process occurs continuously; however, if the process evolves discretely, they involve a difference equation. 
This may happen, for instance, in estimating the size of a future generation based on the size of the current generation.

Let $x_{n+1}=f\left(x_{n}\right)=f^{n+1}\left(x_{0}\right)$ give the size of the $(n+1)$ st-generation as a function of the size of the $n$ th-generation. If 1 is the theoretical maximum for the size of the population, then $f$ is a continuous function on $[0,1]$. The sequence $\left\{f^{n}(x)\right\}$ is called the orbit of $x$. A point $x$ is a periodic point if, for some $n, f^{n}(x)=x$. The first such $n$ is called the period of $x$. The set of subsequential limit points of the sequence $\left\{f^{n}(x)\right\}$ is the $\omega$-limit set of $x$ with respect to $f$ and is denoted by $\omega(x, f)$.

A common difference equation is the logistic difference equation $x_{n+1}=$ $k x_{n}\left(1-x_{n}\right)$. The family of functions $f_{k}(x)=k x(1-x)$ will be called the logistic family. Ideally, the population will stabilize or become semistable, meaning the sequence $x_{0}, x_{1}, x_{2}, \ldots$ will converge to some fixed point or periodic point of the function $f$, and this end behavior will be independent of the initial observed population $x_{0}$. If this does not happen, perhaps a small error in the initial estimate of the size of the population will only have a small effect on the model's estimated values for future generations. However, in many cases, these nice outcomes do not occur. Even with simple models like the logistic family, chaotic behavior can occur. There are many definitions of chaos, but they all describe maps where initial estimates which are close together have orbits which spread out or $\omega$-limit sets that are far apart.

One of the first definitions of chaos to appear in the literature was given by Li and York in a paper entitled "Period Three Implies Chaos" [61]. As one might guess from the title, the authors developed a notion of chaos and then proceeded to show that whenever a function $f:[a, b] \rightarrow[a, b]$ had a point $x \in[a, b]$ so that $x$ had an orbit of period three with respect to $f$, then $f$ behaved chaotically. They deemed a function chaotic if there is a large set of points whose orbits regularly get close together and then far apart.

Definition 1. A set $S$ is scrambled if, for every pair of points $x, y \in S$,

$$
\begin{aligned}
& \limsup _{n \rightarrow \infty}\left|f^{n}(x)-f^{n}(y)\right|>0, \text { and } \\
& \liminf _{n \rightarrow \infty}\left|f^{n}(x)-f^{n}(y)\right|=0 .
\end{aligned}
$$

Definition 2. [61] A function $f:[0,1] \rightarrow \mathbf{R}$ is LY-chaotic if it possesses a scrambled set containing at least two points [61].

$\mathrm{Li}$ and York originally assumed that for each point $x$ in a scrambled set,

$$
\limsup _{n \rightarrow \infty}\left|f^{n}(x)-f^{n}(p)\right|>0,
$$

where $p$ is a periodic point of $f$. The assumption that the orbits of points need to be a certain distance from a periodic orbit is redundant, for only one 
such point is ever in a scrambled set. Originally $\mathrm{Li}$ and York required an uncountable scrambled set. However, in 1986, Smital and Kuchte proved that a two point scrambled set implies the existence of an uncountable scrambled set [59].

The year after Li and York's article, May published a paper describing the dynamics of the logistic family [63]. He shows that higher values of the coefficient $k$ lead to more and more complicated dynamics. For $k>3.57$, the logistic family exhibits LY-chaos. So even functions with simple analytic structure can be chaotic. After the appearence of May's article, mathematical interest in the study of dynamical systems increased and knowledge of a remarkable theorem of Šarkovskii's became more widespread. He put an ordering on the positive integers

$3 \prec 5 \prec 7 \prec \ldots \prec 2 \cdot 3 \prec 2 \cdot 5 \prec \ldots \prec 2^{2} \cdot 3 \prec 2^{2} \cdot 5 \prec \ldots \prec 2^{n} \prec \ldots \prec 2^{2} \prec 2 \prec 1$

and proved:

Theorem 8 (Šarkovskii). [80] If $f$ has an orbit of period $n$, then $f$ has an orbit for each period $m$ with $n \prec m$ in the Šarkovskii's order.

Suppose that $f$ has an orbit of period $m \cdot 2^{k}$ where $m$ is an odd integer. By Šarkovskii's ordering $f$ will also have an orbit of period $3 \cdot 2^{k+1}$. So $f^{2^{k+1}}$ has an orbit of period 3 and a scrambled set by Li and York's results. Li and York show that if $f$ has an iterate that has a scrambled set, then $f$ also has a scrambled set. Hence, we see that if $f$ has an orbit which is not a power of two, then $f$ has a scrambled set and it is LY-chaotic [41].

Block and Copple extended this stratification and used it to develop a useful characterization of chaos that is strictly stronger than LY-chaos. Block and Copple's notion of chaos uses the notion of turbulence.

Definition 3 (Block and Copple). A function is turbulent if there exist intervals $J$ and $K$ with at most one point in common, so that

$$
J \cup K \subset f(J) \cap f(K)
$$

Definition 4. A function is BC-chaotic if some iterate of $f$ is turbulent [12].

Block and Copple show that a function is turbulent only when it has periodic points of all periods, while functions are chaotic whenever they have periodic points with periods that are not powers of 2 . In the proofs of these results, the overlapping of intervals required by their definition of chaos forces the periodic points of odd period to exist as $f$ is iterated.

Another measure for chaos is topological entropy. Topological entropy was first introduced by Adler, Konheim and McAndrew [1] in 1965 as a numerical 
measure for the complexity of an endomorphism of a compact topological space. It is a generalization of measure theoretical entropy which was already being used in this capacity by the Russian school. See $[1$, p. 317] for a definition. In 1979, Misiurewicz [69] showed that a continuous map is BCchaotic if and only if it has positive topological entropy, $h(f)>0$. The easier half of the proof was given by Bowen and Franks [17] in 1976. (We have included a discussion of topological entropy not because we use this notion extensively, but because the language of entropy is used in the literature rather than $\mathrm{BC}-$ chaos. Rather than saying that a function is chaotic in the sense described by Block and Copple, authors will often write $h(f)>0$, even though they use the properties of turbulence to obtain their results.)

Each of the definitions of chaos discussed thus far have derived their definitions from the separation of trajectories of points or the mixing of intervals under iteration. Bruckner and Ceder took a different approach. They considered the class $\mathbf{K}$ of compact subsets of the interval [0,1], furnished with the Hausdorff metric, and studied the map $\omega_{f}: I \rightarrow \mathbf{K}$ defined by $\omega_{f}(x) \rightarrow \omega(x, f)$ [23]. If we look at the class of all $\omega$-limit sets for a given function $f$, calling it $\Omega(f)$, as a subset of $\mathbf{K}$, then $\Omega(f)$ is a closed subset of $\mathbf{K}$ [14].

Applying the restriction that the function $\omega_{f}: I \rightarrow \mathbf{K}$ is continuous is very restrictive, for this implies that each $\omega$-limit set for $f$ is a fixed point or a periodic point with period 2. However, looking at the Baire class of $\omega_{f}$ turns out to be a fruitful measure of chaos. It can be shown that $\omega_{f}$ is always in the second Baire class, $B_{2}$, where $B_{2}$ is the set of functions that can be obtained as pointwise limits of members of $B_{1}$. We will call functions $\mathrm{f}$ with $\omega_{f} \notin B_{1}$, $\omega$-chaotic. Bruckner and Ceder showed that their notion of chaos is strictly intermediate to LY-chaos and BC-chaos [23].

Theorem 9 (Bruckner and Ceder). Let $f \in C(I, I)$. Then

1. $\omega_{f} \in B_{2}$,

2. If $f$ is not $L Y$-chaotic, then $\omega_{f} \in B_{1}$ (but the converse fails),

3. If $\omega_{f} \in B_{1}$, then $f$ is not BC-chaotic (but the converse fails),

Or simply,

$B C-$ Chaos $\Rightarrow \omega-$ chaos $\Rightarrow L Y-$ Chaos.

(It is interesting to note that for the functions from $[0,1]$ into $K$ with the Hausdorff metric, the Borél and Baire classes coincide for finite ordinals. The only thing needed to show this is an extention of the Tietze extension theorem to functions in this class. Namely, if $F_{1}, \ldots, F_{n}$ are mutually disjoint nonempty closed subsets in $[0,1]$, and $Y_{1}, \ldots, Y_{n}$ are in $\mathbf{K}$, then there exists continuous functions $f:[0,1] \rightarrow \mathbf{K}$ such that $f\left(F_{i}\right)=Y_{i}$ for all $i$.) 
For the typical continuous function, $\omega_{f}$ maps each of the points in a residual set of full measure to its own Cantor set [3]. Since all of these points are getting mapped to Cantor sets, which are all contained in a zero measure set, the map $\omega_{f}$ is going to be continuous at these points. Hence, "for the typical continuous function $f$, the map $x \rightarrow \omega(x, f)$ is continuous at the typical point $x$ [60]."

This is not the $\omega$-limit structure that is generally seen when studying analytic functions like those in the logistic family. In fact the $\omega$-limit sets for these types of functions can be described using the language of attractors, a notion closely related to $\omega$-limit set. An attractor is a set which contains the $\omega$-limit sets for a large subset of $I$, and each point of the attractor plays a crucial role. The set of points whose $\omega$-limit set lies within the attractor is called the basin of attraction. When we say that the basin of attraction must be large, we measure large either in terms of Lebesgue measure or Baire Category. We want the attractor to be the smallest set that will have a basin of attraction of the same size. Specifically, we have the following two definitions.

Definition 5. A closed set $A$ is an attractor for $f$ if

1. The realm of attraction, $\rho(A)=\{x: \omega(x, f) \subset A\}$ has positive measure.

2. There is no strictly smaller set $A^{\prime} \subset A$ so that $\lambda(\rho(A))=\lambda\left(\rho\left(A^{\prime}\right)\right)$.

Definition 6. A closed set $A$ is a topological attractor for $f$ if

1. The realm of attraction, $\rho(A)=\{x: \omega(x, f) \subset A\}$ is second category.

2. There is no strictly smaller set $A^{\prime} \subset A$ so that $\rho(A) \backslash \rho\left(A^{\prime}\right)$ is first category.

By considering attractors, we are asking the question, "what is the smallest set we can find for which there a positive probability that the $\omega$-limit set for an arbitrary point chosen from $I$ will be contained in this set?" ¿From the experimental scientists' point of view, this is the logical question, for they are interested in the likelihood of a certain outcome. If we require our function to be smooth in a certain sense, we can predict the type and number of attractors. Researchers discovered that for the unimodal functions that were being studied, most points were being attracted to the same $\omega$-limit set. They called this set the attractor for the function. The attractor always turned out to be a periodic point, a transitive periodic interval, or a Cantor-like set.

The study of iterated maps has a long history. Poincáre made some of the first contributions in the 1880's while studying the iterates of maps of the circle. In the 1910's, Fatou and Julia studied the behavior of iterates of analytic maps [37], [38], and [53]. They showed that if $f$ is a polynomial map, then only a finite number of its periodic points can be topological attractors. In 
particular, Fatou showed that each map in the logistic family $f_{k}(x)=k x(1-x)$ with $k \in[0,4]$ has at most one topological attractor see [67].

Scientists found very complicated dynamics for even these well behaved families of functions [63]. However, it seemed there was always an attractor which attracted almost every and residually many of the points in $I$. Empirical evidence suggests that smoothness restrictions on a function will limit the type and number of attractors; determining these smoothness conditions has stimulated research. Recent papers that relate to this question include [15], [16] and [48]. In particular, the relationship between the topological and measure theoretical attractors is not completely understood. The definitions of attractors referred to are not used consistently throughout the early literature, so it is sometimes difficult to relate different authors' results. With the definition given, mathematicians have shown that the logistic family has unique topological and measure theoretical attractors [16]. This behavior does not occur in every unimodal continuous map, so some feature of the logistic family had to imply this desirable behavior. In 1932, Denjoy introduced the concept of a wandering interval [30].

Definition 7 (Denjoy). An subinterval $J \subset I$ is a wandering interval if each of the successive images of $J, J, f(J), f^{2}(J), \ldots$ are pairwise disjoint.

Maps in the logistic family as well as all piecewise monotone maps don't have wandering intervals, and this is the key to their desirable behavior. In fact, Denjoy constructed a diffeomorphism $f \in C^{1}\left(S^{1}, S^{1}\right)$ of the circle, $S^{1}$, without periodic points and having a wandering interval $J \subset S^{1}$. However, for $C^{2}$ diffeomorphisms of the circle, such wandering intervals cannot exist [30]. In 1963, Schwartz gave another proof of this result [81], but neither Denjoy's or Schwartz's techniques could be applied to the maps of the interval until Singer, introduced the analytical tool called the Schwartzian derivative,

$$
S(f)=\frac{D^{3} f}{D f}-\frac{3}{2}\left(\frac{D^{2} f}{D f}\right)^{2} .
$$

Singer showed that for functions with $S(f)<0$, the basin of any periodic attractor must contain a critical point [83].

In 1979, using this idea and the techniques of Schwartz, Guckenheimer and Misirewicz, Singer proved the nonexistence of wandering intervals for unimodal maps with an everywhere negative Schwartzian derivative [81], [42], and [70]. A map $f \in C^{3}(I, I), f(0)=0=f(1), D f(0)>1$, with unique critical point $c$, and with $S(f)<0$ everywhere except the critical point will be called $S_{-}$ unimodal. In 1985, Milnor used this result to describe the types of attractors of S-unimodal maps of $I$ [68]. (Compare the same result in Preston's book which uses slightly different language [77].) 
Theorem 10 (Milnor). For $S$-unimodal maps there is only one attractor, $A$, and it has one of the following forms:

1. A is a limit cycle i.e. it is the orbit of a periodic point whose basin has nonempty interior.

2. $A=\bigcup_{0}^{p-1} f^{k}(J)$ is the cycle of transitive periodic intervals; ie: $f^{p}(J) \subset$ $J$ and $f^{p} \mid J$ is topologically transitive.

3. $A=\bigcap_{n=1}^{\infty} \bigcup_{k=0}^{p_{n}-1} f^{k}\left(I_{n}\right)$ is a solenoid; here $I_{n}$ is a periodic interval with period $p_{n} \rightarrow \infty, p_{n} \mid p_{n+1}, I_{1} \supset I_{2} \supset \ldots$ We can also describe $A$ as the closure of the orbit of the critical point.

In each case, the basin of attraction is a residual set $B$, with $\omega(x, f)=A$ for each $x \in B$. Furthermore, when $A$ is either a limit set or a solenoid then for almost every point $x \in I, \omega(x, f)=A$.

To see how each of these types of attractors occur in the logistic family read [33]. In general, this is the behavior that a researcher expects to see when he iterates a function. In case 1 , the function may or may not be chaotic. In particular, if the limit cycle has a period of a power of 2 , then the function is not chaotic; if the limit cycle is not a power of 2 , then the function is chaotic. In case 2 , the function is always chaotic. In case 3 the function will be chaotic if it is not a $2^{\infty}$-function. A function $f$ is called a $2^{\infty}$-function if $f$ has a periodic point of period $2^{n}$ for each $n$ and no periodic points with period not a power of 2. This will occur if each of the primes in the solenoid is 2 . When this happens any of the three types of chaos may be possible and the distinction between the types of chaos can be seen. Bruckner and Ceder describe what the $\omega$-limit sets, and therefore the attractor in this case, must look like for a function to be chaotic in each of the three senses decribed above [23].

There are several $\omega$-limit set structures which define chaos. A set $M \subset I$ is a minimal set with respect to $f$ if $f(M)=M$ and $M$ has no proper subset which is non-empty and invariant relative to $f$. Notice that $\omega$-limit sets are invarient under $f$. A point $x$ is a homoclinic point if there is a periodic point $z$ with $z \in \omega(x, f)$ and $x \in \bigcap_{\epsilon>0} \bigcup_{m \geq 0} f^{m}(z-\epsilon, z+\epsilon)$. That is, for each neighborhood $U(z, \epsilon)$ of $z$ there is an $m$ so that $x \in f^{m}(U(z, \epsilon))$. See $[12$, p.153]. Thus, a homoclinic point is one that tends towards a periodic point under backward iteration and which lands on the same periodic point under forward iteration. When $f$ has a homoclinic point $x$, with an associated periodic point $z$, then in every neighborhood of $z$, there are infinitely many distinct periodic points [33, p.125]. The set of all periodic points is denoted 
by $\operatorname{Per}(f)$. We get the following equivalences by compiling the results from several authors [79], [69], [11], [84].

Theorem 11. The following are equivalent for each $f \in C$ :

1. $f$ is not BC-chaotic,

2. $f$ has zero topological entropy, $h(f)=0$,

3. The period of every periodic orbit is a power of 2 ,

4. There are no homoclinic trajectories,

5. The set of all periodic points of $f, \operatorname{Per}(f)$, is a $G_{\delta}$,

6. Every $\omega$-limit set contains a unique minimal set, and

7. If $\omega(x, f)$ is infinite, then $\omega(x, f) \cap \operatorname{Per}(f)=\emptyset$.

If $f$ is not LY-Chaotic or $\omega$-chaotic, then each of the properties (1)-(7) hold. However, more can be said in the case of non-LY-chaos. In particular, the $\omega$-limit sets for $f$ will either be finite periodic orbits or Cantor sets. There is a slight difference between the $\omega$-limit sets for LY-chaotic functions and those for $\omega$-chaotic functions. Namely, a $2^{\infty}$-function is $\omega$-chaotic if and only if it has an infinite $\omega$-limit set with isolated points [23]. Functions with this property are LY-chaotic, but not every LY-chaotic function has an infinite $\omega$-limit set with isolated points. A function will be LY-chaotic if and only if there is not a periodic point between every two points of any infinite $\omega$-limit set. Functions which are not LY-chaotic also have nice properties with respect to the set of all $\omega$-limit points, $\omega(f)=\cup\{\omega(x, f): x \in[0,1]\}$. Gongfu showed that $\omega(f)=\left\{x \in[0,1]: \lim _{n \rightarrow \infty} f^{2^{n}}(x)=x\right\}$ whenever $f$ is not LY-chaotic [40].

We will now contrast the $\omega$-limit sets of smooth, nonchaotic functions with the behavior of the typical continuous function. Usually, typical continuous functions exhibit considerable pathology in comparison with well behaved functions; the same is true with respect to iteration. It is rather surprising that we can say anything about the iterative behavior of a typical continuous function considering the difficulties encountered when studying 'nice' functions. However, the regularity seen in the intersecton patterns of a typical continuous function also appears in the study of their iterative behavior. Much of the work done in this area can be found in Dynamics of Typical Continuous Functions by Agronsky, Bruckner and Laczkovich [3]. They start by describing the $\omega$-limit structure of a typical continuous function, obtaining several interesting results along the way. They finish by describing how a typical point of a typical continuous function behaves. They use the standard method of 
showing that certain subsets of $C$, with the properties we will describe below, are residual in $C$.

The next theorem provides a contrast between the behavior of the typical continuous function and the behavior of the classes of 'nice' continuous functions with respect to smoothness and chaos that we discussed above. Instead of one or finitely many attractors there are none, because all of the action is taking place on a null set. Most $x$ have their own $\omega$-limit set, which turns out to be a Cantor set [3].

Theorem 12 (Agronsky, Bruckner and Laczkovich). The typical continuous function, $f$ has the following properties:

1. For each $x$ in some residual subset of $[0,1]$ there corresponds a Cantor set $H=\omega(x, f)$.

2. There are c pairwise disjoint such Cantor $\omega$-limit sets, where $c$ is the cardinality of the continuum.

3. If $H$ is such a Cantor $\omega$-limit set, then $f$ maps $H$ homeomorphically onto itself and each $x \in H$ has a dense orbit in $H$.

4. The basin of attraction of $H, \rho(H)=\{x: \omega(x, f) \subset H\}$, is nowhere dense.

In the process of proving this result, the authors discover that for a typical continuous function $f \in C$ every neighborhood of every periodic point contains periodic points of arbitrarily large periods. Simon proceeded to make this result more specific by showing that in the neighborhood of every periodic point of period $n$ there are periodic points of period $n \cdot k$ for every integer $k$ [82]. From this we can see that the usual notion of an attractive periodic orbit for such functions is impossible. Further, for each $f$ in this residual set and for each sequence of intervals $\left\{J, f(J), \ldots f^{n-1}(J)\right\}, \bigcup_{m=0}^{\infty} f^{m}(J)$ contains a Cantor set and is thus contained in a nondegenerate interval. Thus, an interval cannot contract about a point as one finds in the simple attracting periodic orbits for the logistic family.

For a typical continuous function, no point has a dense orbit in any interval. Suppose that the orbit of $x, \gamma(x, f)=\left\{f^{n}(x): n=1,2,3 \ldots\right\}$ is dense in an interval $J$. Then $E=\{x \in J: \gamma(x, f)$ is dense in $J\}$ would be dense in $J$ and of type $G_{\delta}$ and hence residual. But then no point of $E$ could be attracted to a Cantor set, which would violate part (1) of the theorem. Thus the transitive periodic intervals that are attractors for the S-unimodal do not occur for a typical continuous function.

A typical function $f$ has no dense orbits. If we iterate a typical point $x$ in $I$ the sequence $g^{k}(x)$ will appear to move randomly for a while until it enters its 
microscopic attraction to its Cantor set $K$. The set $K$ may be contained in a very small interval in which case it will appear to 'the scientist' that the orbit approaches a fixed point. If the smallest interval containing $K$ can be 'seen', then 'the scientist' will observe periodic motion with the period depending on the accuracy of the computer. It may also be that it takes so long for the orbit of $x$ to be attracted to $K$ that the limiting behavior is never observed, giving the appearance of random behavior and the transitive periodic interval that the 'scientist' expects to see. For more on the analysis of this discussion see [3].

Now, for any subset $K \subset I$ and $f \in C$ let

$$
K_{f}^{*}=\bigcup_{n=-\infty}^{\infty} f^{n}(K) \quad A(f)=\bigcup_{x \in I} \omega(x, f) .
$$

Theorem 13 (Agronsky, Bruckner and Laczkovich). Let $K \subset[0,1]$ be of first category and full measure. For $f \in C$, let $L_{f}=I-K_{f}^{*}$. Then for the typical function $f$ in $C$ :

1. $A(f) \subset L_{f}$ (hence $A(f)$ is of measure zero),

2. $\omega(x, f)$ is perfect for every $x \in K_{f}^{*}$,

3. $\omega(x, f) \cap \omega(y, f)=\emptyset$ for every $x, y \in K, x \neq y$, and

4. $f$ is one-to-one on $\bigcup_{n=0}^{\infty} f^{n}(K)$.

This theorem gives a lot of information about the iterative behavior of the typical continuous function. A typical point in $[0,1]$ is attracted to a Cantor set and we may prescribe any residual subset of $[0,1]$ to contain the closure of the union of all $\omega$-limit sets for all $f$ in a residual subset of $C$. Within the proof we find that for a typical continuous function $f$, the set of fixed points of $f^{n}, \operatorname{Fix}\left(f^{n}\right)$, is nowhere dense and perfect. Simons shows that this set is actually bilaterally strongly $\sigma-$ porous [82]. He gets as corollaries for typical continuous functions $f$,

1. The set of periodic points of period $\mathrm{k}$ is dense and relatively open in $\operatorname{Fix}\left(f^{k}\right)$,

2. The set of periodic points of period $\mathrm{k}$ is dense in itself, and

3. The set of periodic points of period $\mathrm{k}$ is not closed for every $k>1$.

We see that the typical continuous function does not have an attractor. $A(f)$ does contain the $\omega$-limit sets of a full measure subset of $I$. However, no set has $\omega(x, f)$ equal to $A(f)$. In fact a subset of $A(f), A(f)-H$ for any of the 
Cantor $\omega$-limit sets $H$, also attracts a subset of $I$ of full measure. Thus, $A(f)$ does not satisfy the second criterion for an attractor. No set is an $\omega$-limit set for any set of positive measure as in the case of nicely behaved functions. There are no periodic orbits, periodic transitive intervals, or solenoids which attract a set of positive measure.

Mathematicians sometimes put added restrictions on continuous functions like monotonicity, differentiability or analyticity to get the behavior that they desired. Similarly, mathematicians studying iteration of continuous functions had to put additional restrictions on the functions they were studying in order to get the results that they desired. One might wonder what the $\omega$-limit sets for a typical differentiable function or continuously differentiable function might look like.

Bruckner and Steele approached the behavior of functions under iteration from a different vantage point. Rather than looking at how the Baire Category Theorem applied to the space of continuous functions $C$ they look at the space $\mathcal{K}$ of closed nonempty subsets of the unit interval with the Hausdorff metric. The Hausdorff metric on $\mathcal{K}$ is given by

$$
d(E, F)=\inf \left\{r: E \subset V_{r}(F) \text { and } F \subset V_{r}(E)\right\}
$$

where $V_{r}(C)=\{y \in[0,1]: \operatorname{dist}(y, C)<r\}$. In the process of trying to understand $\omega$-limit sets of continuous functions they studied the typical element of this complete metric space. They found that the typical elements of $\mathcal{K}$ are small Cantor sets which can also be taken to be subsets of the irrationals. Let $\mathcal{K}^{*}$ represent this large subset of $\mathcal{K}$. We can also leave out the set

$$
\mathcal{A}=\left\{E \in \mathcal{K}: \begin{array}{l}
\text { there are disjoint portions } P \text { and } Q \text { of } E \\
\text { and } f \text { Lipschitz such that } f(P) \supset Q
\end{array}\right\} .
$$

Giving us $\mathcal{K}^{*}-\mathcal{A}$ as all but a first category subset of $\mathcal{K}[27]$.

It has been shown that every nonempty nowhere dense closed set is an $\omega-$ limit set for some continuous function. However, the construction produces a highly non-smooth function [2]. Given the typical $\omega$-limit set in $\mathcal{K}$, one might wonder how smooth can the function with this $\omega$-limit set be. Bruckner and Steele have shown that we cannot even assume that the function with the typical $\omega$-limit set is Lipschitz [27]. Since $\omega$-limit sets map into themselves upon iteration, it makes sense to study the behavior of functions that map a generic element $E \in \mathcal{K}$ into itself. They show that unless $f$ is the identity on some portion of $E$, there is a Cantor subset $D \subset E$ on which $f$ is nowhere Lipschitz. The set $D$ may be nowhere dense in $E$, but it is large in the sense that it maps onto all of $E$ while its complement in $E$ maps onto a first category subset in E. It then follows that $f$ is nondifferentiable on a dense subset of $D$ of type $G_{\delta}$, and that $f$ maps its set of points of differentiability in $E$ onto a first category subset of $E$. Thus, if $E \in \mathcal{K}^{*}-\mathcal{A}$ and is an $\omega$-limit set for 
$f$, then $f: E \rightarrow E$. Suppose that $f$ is not the identity on any portion of $E$, then $f$ cannot be Lipschitz on $I$ for there is a Cantor set $K \subset E$ such that $f \mid K$ is not Lipschitz on any portion of $K$. Furthermore, $f$ is not differentiable on a dense $G_{\delta}$ subset of $K$. It follows that the generic closed set $E$ is not an $\omega$-limit set for any Lipschitz function or any differentiable function.

\section{Kolmogorov's Theorem}

How complicated can a continuous function be? Certainly the examples of nowhere differentiable functions, the intersection patterns, and the $\omega$-limit behavior of the typical continuous functions make us believe that functions $f \in C[I, \mathbf{R}]$ can be badly behaved. What happens if we look at continuous functions of several variables, $f \in C\left[I^{n}, \mathbf{R}\right]$ ? Does the possible behavior become more complex? Sprecher states that, "the increase in the degrees of freedom with increasing $n$ does not increase the functional complexity, thereby implying that the "worst" functions of $C[I, \mathbf{R}]$ are as 'bad' as the 'worst' functions of $C\left[I^{n}, \mathbf{R}\right][86]$. The meaning of the above statement as well as its proof are related to Baire's category theory. Most of the following development is taken from a series of expository articles by Sprecher and Lorentz [85], [86], [62].

Hilbert conjectured in his thirteenth problem presented in his famous talk at the International Congress of Mathematics in Paris in 1900 that, "the equation of the seventh degree $f^{7}+x f^{3}+y f^{2}+z f+1=0$ is not solvable with the help of any continuous functions of only two variables [45]." The stated function of the seventh degree is general in the sense that it can be obtained from an arbitrary seventh degree polynomial $\phi(f)=\sum_{i=1}^{7} a_{1} f^{i}$ by a series of algebraic equalities involving functions that use only one variable.

The statement of this problem originated out of the attempts to eliminate by algebraic means the largest possible number of coefficients from polynomial equations $\sum_{k=0}^{n} a_{k} x^{k}=0$, thereby expressing their solutions, regarded as functions of $n+1$ coefficients as functions of fewer coefficients.

It was Hilbert's notion that the number of variables of a function is a useful measure of functional complexity in the following sense: Consider a continuous function $f$ of $n$ variables, and a class $S_{m}$ of continuous functions of $m \leq n$ variables. If the function $f$ can be represented as a finite number of sums and superpositions of functions $S_{m}$, and if $m$ is the least natural number for which this is true, then we can use $m$ as a measure of the functional complexity of $f$ relative to the class $S_{m}[86]$.

Here, a superposition means a composition of functions. For instance, let 
$f(x, y)=x y$. We can write this in terms of a finite number of superpositions of continuous functions of one variable and the binary operation of addition. This follows from the observation that

$$
x y=\frac{1}{4}(x+y)^{2}-\frac{1}{4}(x-y)^{2}=u([a(x)+b(y)])+v([c(x)+d(y)])
$$

where $u(t)=-v(t)=\frac{1}{4} t^{2}$ and $a(t)=b(t)=c(t)=-d(t)=t$ are all functions of one variable. Kolmogorov showed that this constructionis possible for all continuous functions of any number of variables. An improvement of Kolmogorov's theorem, given by Hedberg, [44] can be stated as follows.

Theorem 14 (Hedberg). If $n \geq 2$ there are real numbers $\lambda_{1}, \lambda_{2}, \ldots, \lambda_{n}$ and continuous functions $\phi_{1}, \ldots, \phi_{2 n+1} \in C[I]$ with the following property: for each continuous function $f \in C\left[I^{n}, \mathbf{R}\right]$ there exists $g \in C[\mathbf{R}]$ such that

$$
f\left(x_{1}, \ldots, x_{n}\right)=\sum_{k=1}^{2 n+1} g\left(\lambda_{1} \phi_{k}\left(x_{1}\right)+\cdots+\lambda_{n} \phi_{k}\left(x_{n}\right)\right)
$$

In this case, $h_{k}\left(x_{1}, \ldots, x_{n}\right)=\lambda_{1} \phi_{k}\left(x_{1}\right)+\cdots+\lambda_{n} \phi_{k}\left(x_{n}\right)$ is the sum of functions of one variable, $g$ is a function of one real variable, and $f$ is the sum of $2 n+1$ functional values of $g$. In other words, any continuous function of $n$ variables can be written as a superposition of continuous functions of a single variable. Thus, Hilbert's measure of functional complexity does not distinguish between classes of continuous functions of different numbers of variables. It is from this theorem that Sprecher made the observation that the 'worst' continuous functions of one variable are as 'bad' as the 'worst' continuous functions of several variables.

The category method can be employed to prove Kolmogorov's theorem. The proof Kolmogorov gives of his theorem is quite technical; using the category method eliminates many complicated constructions. In addition, the category method phrases Kolmogorov's theorem in a somewhat more general setting. Namely, $\lambda_{1}, \lambda_{2}, \ldots, \lambda_{n}$ can be chosen so that all of the $(2 n+1)$-tuples of continuous functions $\left(\phi_{1}, \ldots, \phi_{2 n+1}\right)$, except a set of first category relative to the metric space $C(I)^{2 n+1}$, have the stated property [44]. The number of summands, $2 n+1$, is the best possible. Doss gave a proof for $n=2$ in 1963, but the general case was not proved until 1983 when Sternfeld published an extensive paper giving insight into Kolmogorov's theorem [87].

Kolmogorov's theorem does not hold when we put certain smoothness conditions on the function in addition to continuity. We know that smoothness conditions provide a useful measure of functional complexity. For instance, there is an analytic function of three variables that cannot be represented as a finite superposition of analytic functions of two variables [89]. The earliest 
results along these lines were obtained by Ostrovski in 1920 [74]. He proved that the analytic function

$$
\zeta(x, y)=\sum_{n=1}^{\infty} \frac{x^{n}}{n^{y}}
$$

cannot be represented as a finite superposition of infinitely differentiable functions of one variable and algebraic functions of any number of variables.

Vituskin developed a functional to determine the complexity of a function which took into account both smoothness conditions and number of variables. Unlike Hilbert's conjecture, this functional could distinguish functions with differing numbers of variables as follows: Let $q=p+a$ where $p$ is a nonnegative integer and $0<a \leq 1$. Let $W_{q}^{n}$ be the class of $p$-times continuously differentiable functions of $n$ variables defined on a closed bounded region of $\mathbf{R}^{\mathrm{n}}$, whose $p$ th partial derivatives belong to class Lip $(\alpha)$. If we use $n / q$, with $q \geq 0$, as a measure of the functional complexity of the class $W_{q}^{n}$, then not all functions with complexity index $n / q$ can be represented by superpositions of functions with complexity $n^{\prime} / q^{\prime}<n / q$. For instance, this result says that there are functions $f \in C\left[I^{3}, \mathbf{R}\right]$ which cannot be represented as a superposition of continuously differentiable functions of two variables [89]. This is a result that is close to the one that Hilbert was looking for.

\section{Pseudo-arcs and subsets of the plane}

The category method has been used in the space of compact subsets of the plane $\mathcal{K}$, with the Hausdorff metric to determine what typical compact connected subsets of the plane look like. Complicated constructions of curves that do not contain arcs started to appear at the beginning of the twentieth century. At the International Mathematics Conference in Cambridge in 1912, Janiszewski gave a rather sketchy definition of a curve on the plane which contains no arcs. (An arc is any set homeomorphic to an interval, while any one dimensional continuum, or compact connected Hausdorff-space, is a curve.) "At that time, the existence of a curve of that kind seemed quite extraordinary [58, p. 30]." As with the case of the nowhere monotonic differentiable function, the Baire Category Theorem provides and easy proof of existence when constructions are difficult to visualize.

The typical continuum in the plane is a Janiszewski curve or pseudo-arc. It can be shown that a residual set (in fact a dense $G_{\delta}$ ) of curves in the plane are pseudo-arcs, and a pseudo-arc does not contain an arc [8]. A pseudo-arc is defined to be a snakelike, hereditarily indecomposable continuum. A finite sequence of open sets $G_{i}$ is an $\epsilon$-chain of open sets if $G_{i} \cap G_{j} \neq \emptyset$ if and only if $|i-j| \leq 1$ (the $G_{i}$ intersect each other only if they are adjacent) and the 
diameter of each $G_{i}$ is less than $\epsilon$. A set is snakelike if for each $\epsilon>0$ there is a chain of open sets $G_{i}$ containing the set. Certainly, an arc is snakelike, but arcs are decomposable. A continuum is indecomposable if it cannot be written as the union of two proper subcontinua. If $P$ is any point in the interior of the arc $A B$ then the arcs $A P$ and $P B$ are proper subcontinua such that their union is all of $A B$, making the arc $A B$ decomposable. Finally, a property is hereditary if it is enjoyed by every nondegenerate subspace. In our case, this means that not only is a pseudo-arc indecomposable, each of its subspaces are indecomposable. Every two pseudo-arcs are homeomorphic [71]. Thus, like an arc, a pseudo-arc is homeomorphic to each of its subcontinua. Prior to Moise's paper the only known homogeneous nondegenerate locally connected plane continuum was the simple closed curve. In 1921, Mazurkiewicz had asked whether any continua other than an arc had the property that is was homeomorphic to each of it's subcontinua [65] [58, vol. II, p.225].

To get a picture of what a Pseudo-arc and an indecomposable continuum look like we need a theorem by Mazurkiewicz in 1927 [66, p.305]. A space is irreduciblebetween the points $a$ and $b$, provided it is connected and these two points cannot be joined by any closed connected set which is different from the whole space [58, vol. II, p.190].

Theorem 15 (Mazurkiewicz). A complete space $X$ is indecomposable if and only if $X$ contains three points $a, b$, and $c$ between every pair of which it is irreducible.

This theorem suggests an example of a snakelike indecomposable continuum, $K$, described as follows: Start with three points, $a, b, c$, in $\mathbf{R}^{2}$. For $n=0,1,2, \ldots$,

let $C_{0}$ be a chain from $a$ to $c$ through $b$,

let $C_{3 n+1}$ be a chain from $b$ to $c$ through $a$,

let $C_{3 n+2}$ be a chain from $a$ to $b$ through $c$,

let $C_{3 n+3}$ be a chain from $a$ to $c$ through $b$.

In each case, $C_{k} \subset C_{k+1}$. Then

$$
K=\bigcap C_{k}=\bigcap C_{3 n}=\bigcap C_{3 n+1}=\bigcap C_{3 n+2} .
$$

But, $\bigcap C_{3 n}$ is irreducible between $a$ and $c, \bigcap C_{3 n+1}$ is irreducible between $b$ and $c$, and $\bigcap C_{3 n+2}$ is irreducible between $a$ and $b$. Since the continua is irreducible between each pair of points, it is indecomposable by Mazurkiewicz's theorem. Since each iteration places the continua in a smaller chain, it is snakelike. We can see the first three steps in this iterative process in the picture below [47, p.141]. 


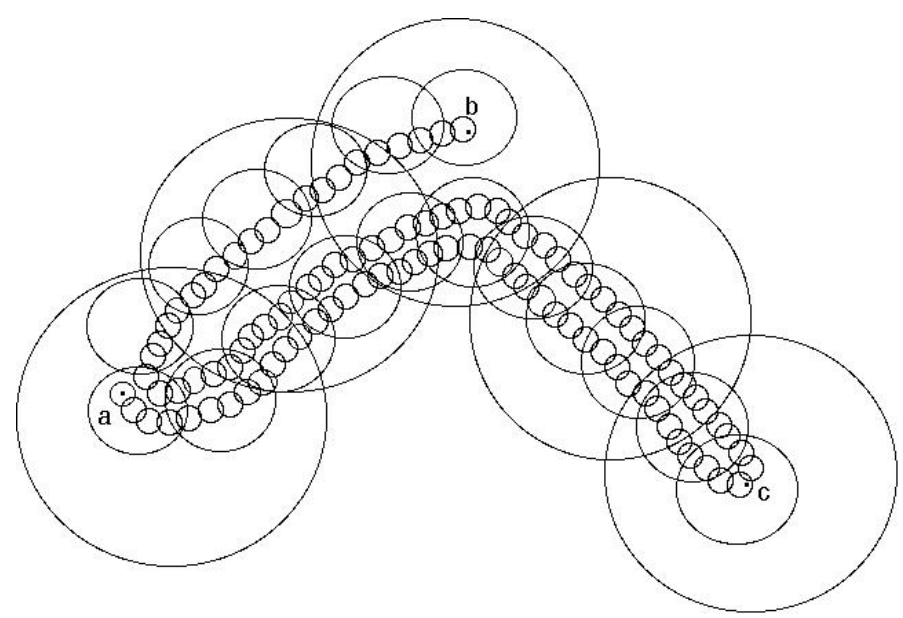

Figure 1. An Indecomposable Continuum

We now have a snakelike indecomposable continuum, but a pseudo-arc has the additional property of being hereditarily indecomposable. We will construct a continuum so that each portion has three points, between each two of which it is indecomposable. The following description was given by Bing [9], the picture appears in Hocking and Young [47, p. 141], and the originators of this construction are Mö̈see [71] and Knaster [56]. If the chain $E=\left[e_{1}, e_{2}, \ldots, e_{h}\right]$ is a refinement of the chain $D=\left[d_{1}, d_{2}, \ldots, d_{m}\right]$, then $E$ is crooked in $D$ provided that if $k-n>2$ and there are links $e_{i}$ and $e_{j}$ of $E$ with $e_{i} \subset d_{n}$ and $e_{j} \subset d_{k}$, then there are links $e_{r}$ and $e_{s}$ of $E$ with $e_{r} \subset d_{k-1}$ and $e_{s} \subset d_{n+1}$ and $i>r>s>j$ or $i<r<s<j$. Figure 2 shows that $E$ is crooked in $D$.

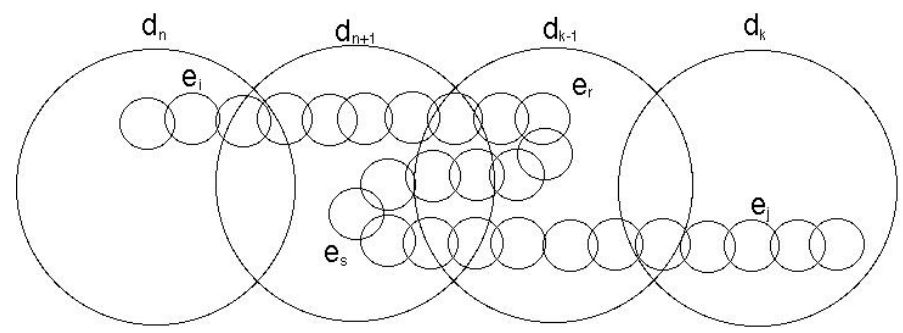

Figure 2. $E$ is crooked in $D$

In the plane let $D_{1}, D_{2}, \ldots$ be a sequence of chains between the distinct points $a$ and $b$ such that for each $i$ :

1. $D_{i+1}$ is crooked in $D_{i}$, 
2. No link of $D_{i}$ has a diameter more than $\frac{1}{i}$, and

3. The closure of each link of $D_{i+1}$ is a compact subset of a link of $D_{i}$.

Then, $D=\bigcap D_{i}$ is a pseudo-arc; that is a snakelike hereditarily indecomposable continuum. The following picture depicts a pseudo-arc. Each $D_{i}$ is a union of links $U_{i, j}$ and pictured in Figure 3 [47, p. 142]. Notice that $D_{1}$ is made from the five links $U_{1,1}, . ., U_{1,5}$ with $\bar{U}_{1,1}$ meeting only $\bar{U}_{1,2}$, and $\bar{U}_{1,2}$ meeting only $\bar{U}_{1,3}$, ect., and $a$ is in $U_{1,1}$ and $b$ ia in $U_{1,5}$. Next there are 45 open sets $U_{2,1}, \ldots U_{2,45}$ as pictured. Any number of subdivisions larger than 4 would have worked to form a pseudo-arc.

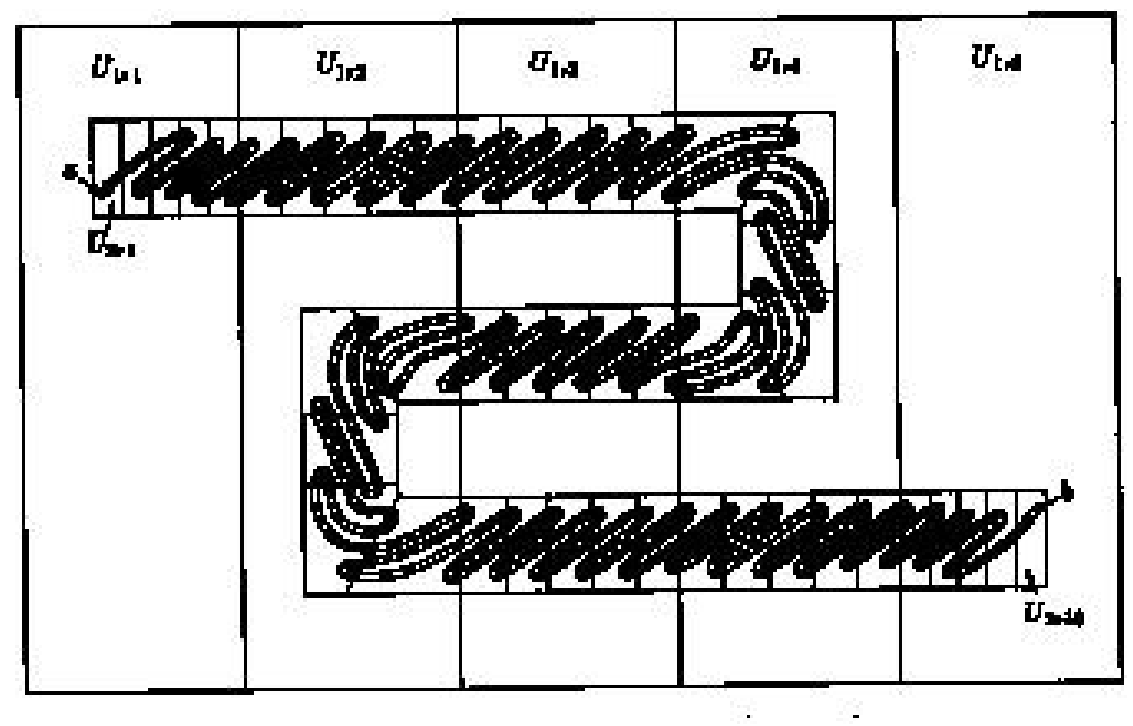

Figure 3. A Pseudo-arc

Earlier we stated that the typical continuum is a pseudo-arc. Bing gave a simple proof for this result using the Baire Category Theorem.

Theorem 16 (Bing). If $X$ is Euclidean n-space or any Hilbert space, then the typical compact continua is a pseudo-arc.

Proof:We want to show that the set of all pseudo-arcs, nondegenerate, snakelike, hereditarily indecomposable continua, form a residual subset of the space of all compact continua with the Hausdorff metric, $\mathcal{K}$. Let $P$ denote the set of all pseudo-arcs, $N$ 
the set of all nondegenerate continua, $S$ the set of all snakelike continua, and $H$ the set of hereditarily indecomposable continua. Then, $P=N \cap S \cap H$. We will show that $P$ is dense and each of $N, S, H$ can be written as a countable intersection of open sets.

Notice first that $N$ is open because the collection of all singletons $\{x: x \in X\}$ is a closed subset of $\mathcal{K}$.

Let $F_{i}$ be the collection of all continua $f \in \mathcal{K}$ such that $f$ cannot be covered by a $1 / i-$ chain. If $\left\{f_{j}\right\}$ is a sequence of continua in $F_{i}$ such that $f_{j} \rightarrow f_{0}, f_{0}$ is also an element of $F_{i}$, for if a $1 / i$-chain covers $f_{0}$ it will also cover $f_{j}$ for some $j$. Hence, $F_{i}$ is closed. Note that $\bigcup F_{i}$ is the set of all nonchainable continua, making $S=\bigcap F_{i}^{c}$ a countable intersection of open sets.

Let $G_{i}$ be the set of all compact continua, $g$, such that $g$ contains a subcontinua $g^{\prime}$ which is the union of two subcontinua $g_{1}$ and $g_{2}$ such that $g_{1}$ contains a point at a distance $1 / i$ or more from $g_{2}$ and $g_{2}$ contains a point at a distance $1 / i$ or more from $g_{1}$. One can see that $G_{i}$ is closed in $\mathcal{K}$, and each element of $\mathcal{K}$ not in $\bigcup G_{i}$ is a hereditarily indecomposable continua. We have $H=\bigcap G_{i}^{c}$ is the intersection of a sequence of open sets, and $P=N \cap S \cap H$ is also a countable intersections of open sets.

To finish showing that $P$ is residual (in fact a dense $G_{\delta}$ ) we need only show that $P$ is dense. Let $\epsilon>0$ and $g \in \mathcal{K}$. There is a broken line $a b$ whose distance from $g$ is less than $\epsilon / 2$. Let $D$ be an $\epsilon / 2$-chain form $a$ to $b$ covering the arc $a b$. Each element of $D$ is the interior of a sphere. With a construction similar to the one given above we can find a pseudo-arc containing $a$ and $b$, which is covered by $D$. The Hausdorff distance between $g$ and $h$ is less than $\epsilon$.

Here the Baire Category Theorem gives an easy proof of what a typical one dimensional subset of the plane looks like. A construction called the Lakes of Wada is an example of a bounded one dimensional continuum which is the boundary of three disjoint domains. This example is explained in Hocking and Young [47, p.143] and is due to Yoneyama [92]. For more information on Pseudo-arcs see [58, vol. II, p. 213] or [47, p.141].

A snakelike continua becomes circlelike if the $a$ coincides with $b$ and the first covering circle only meets the last covering circle and the second covering circle. We can then make the definition of a pseudo-circle to be a hereditarily indecomposable circlelike continuum. This is just the obvious extension of a pseudo-arc. In 1982, M. Handel gave an example of a $C^{\infty}$-diffeomorphism of the plane with a strange attractor that is a pseudo-circle [43]. Thus these bizarre objects in space are examples of the attactors that we looked at earlier. 
M. Herman gave a modification of Handel's construction. He constructed a $C^{\infty}$-diffeomorphism $f$ of the plane with an invariant pseudo-circle, $\Gamma$, which divides the plane into two regions such that $f$ is analytic on the bounded components of $\mathbf{R}^{2}-\Gamma$ [44]. The above two facts can be found in [54, p.310]. In 1995, Kennedy and Yorke gave an example of a $C^{\infty}$-diffeomorphism of a 7-manifold which has an invariant set that contains many connected components that are pseudo-circles. Compare this to the typical $\omega$-limit sets for a continuous function on an interval. Notice that the set which contains all of the $\omega$-limit sets is invariant. Kennedy and York's example is perturbable in the sense that for $C^{1}$ maps sufficiently close to the example map the invariant set again contains uncountably many connected components most of which are pseudo-circles. In fact, $f$ has the following features:

1. There exists an invariant set $\mathbf{B}_{f}$, and a neighborhood $U$ of $\mathbf{B}_{f}$ such that if $f^{n}(x) \in U$ for all integers $n$, then $x$ is in $\mathbf{B}_{f}$.

2. There is a point in $\mathbf{B}_{f}$ with a dense trajectory under $f$.

3. The number of connected components of $\mathbf{B}_{f}$ is uncountable and residually many of the components are pseudo-circles.

4. For each $g$ in some $C^{1}$ neighborhood of $f$, the invariant set $\mathbf{B}_{g}$ has the same structure as $\mathbf{B}_{f}$.

The structure of this invariant set resembles the level set structure of the typical continuous function in section 4 and the typical $\omega$-limit set we saw in section 5 .

Question 1. What do the typical $\omega$-limit sets for continuous functions defined on the plane look like.

Interest in what happens typically continues to be studied for continuous functions. For example, see Descriptive mapping properties of typical continuous functions, by Bernd Kirchheim [55], and Typical continuous functions are virtually nonmonotone, by P. D. Humke and M. Laczkovich [51]. Baire's theorem is increasingly being used in other spaces. See for instance, Points of nondifferentiability of typical Lipschitz functions by D. Preiss and J. Tišer [78].

Some of the questions that the Baire category theorem has helped to address are: what exactly are the implications of continuity to intersection patterns, differentiability, and $\omega$-limit sets? What does the intersection pattern of a continuous function with a line look like? What is the difference between $C^{\infty}$ and analytic? What can subsets of the plane look like? Are continuous 
functions defined on spaces of higher dimension more complex? More references, information, and background for all of these topics can be found in my thesis, as well as more background and applications involving the Baire Category Theorem [52].

\section{References}

[1] R. L. Adler, A. G. Konheim, and M. H. McAndrew, Topological entropy, Trans. Amer. Math. Soc. 114 (1965), 309-319

[2] S. Agronsky, A. M. Bruckner, J. G. Ceder, and T. L. Pearson, The structure of $\omega$-limit sets for continuous functions, Real Anal. Exchange $\mathbf{1 5}$ (1989-90) 483-510

[3] S. J. Agronsky, A. M. Bruckner, and M. Laczovich, Dynamics of typical continuous functions, J. London Math. Soc., 40 (1989), no. 2, 227-243.

[4] R. Baire, Sur les fonctions de variables réelles, Annali di Mat. Pura ed applicata, 3 (1899), no. 3, 1-123.

[5] S. Banach, Über die Baire'sche Kategorie gewisser Funktionenmengen, Studia Math., 3 (1931), 174-179.

[6] S. Banach, Théorie des opérations linéares, Monogr. Mat. WarszawaLwów (1932).

[7] A. S. Besicovitch, A problem on topological transformations of the plane, Fund. Math., 28 (1935), 61-65.

[8] R.H. Bing, A homogeneous indecomposable plane continuum, Duke Math. J., 15 (1948), 729-742.

[9] R.H. Bing, Concerning hereditarily indecomposable continua, Pacific Journal Math., 1 (1951), 43-51.

[10] L. S. Block, Stability of periodic points in the theorem of Šarkovskii, Proc. Amer. Math. Soc., 81 (1981), no. 2, 333-336.

[11] L. S. Block and W. A. Coppel, Stratification of the continuous maps of the interval, Trans. Amer. Math. Soc., 297 (1986), no. 2, 587-604.

[12] L. S. Block and W. A. Coppel, Dynamics in One dimension, Lecture Notes in Methematics, 1513 Springer, Berlin (1992).

[13] A.M. Blokh, The set of all iterates is nowhere dense in $C([0,1],[0,1])$, Trans. Amer. Math. Soc., 333 (1992), no. 2, 787-798. 
[14] A.M. Blokh, A.M. Bruckner, P.D. Humke, and J. Smítal, The space of $\omega-$ limit sets of a continuous map of the interval, MSRI preprint no. 063-94 (1994).

[15] A.M. Blokh and M. Yu. Lyubich, Absence of wandering intervals in one dimensional smooth dynamical systems, Soviet Math. Dokl., 39 (1989), no. $1,169-172$.

[16] A.M. Blokh and M. Yu. Lyubich, Attractors of maps of the interval, Func. Anal. Appl.(Kharkov), 21 (1987), 70-71.

[17] R. Bowen and J. Franks, The periodic points of the maps of the disk and the interval, Topology 15 (1976), 337-342

[18] David Bressoud, A Radical Approach to Real Analysis, The Mathematical Association of America, (1994).

[19] A. M. Bruckner, Differentiation of real functions [2nd ed.] (CRM Monograph Series; v. 5), American Mathematical Society, 1994.

[20] A. M. Bruckner, Current Trends in differentiation theory, Real Anal. Exch. 5 no.1 (1979/80) 9-60.

[21] A. M. Bruckner, J. B. Bruckner, and B. S. Thomson, Real Analysis, Prentice-Hall, 1994.

[22] A. M. Bruckner and Z. Buczolich, Attractive properties via generalized derivatives of continuous functions, Czexhoslovak Math. J., 42 no.117 (1992), Praha.

[23] A.M. Bruckner and J. Ceder, Chaos in terms of the map $x \rightarrow \omega(x, f)$, Pacific J. of Math., 156 no. 1 (1992), 63-96.

[24] A. M. Bruckner and K. Garg, The level set structure of a residual set of continuous functions, Trans. Amer. Math. Soc., 232 (1977), 307-321.

[25] A. M. Bruckner and J. Haussermann, Strong porosity features of typical continuous functions, Acta Math. Hung., 45 (1985), 7-13.

[26] A. M. Bruckner and S. H. Jones, The behavior of continuous functions with respect to intersection patterns, Real Anal. Exch., 19 no. 2 (199394), 414-432.

[27] A.M. Bruckner and T. H. Steele, The lipschitz structure of continuous self-maps of generic compact sets, J. of Math. Analysis and Appl., 188, no. $3,(1994), 798-808$. 
[28] G. Cantor, "Uber unendliche lineare Punktmannichfaltigkeiten, I, II, III, $I V, V$, Mathematische Annalen, 15, 17, 20, 21, 21 (1879, 80, 82, 83, 83), 1-7, 355-358, 113-121, 51-58, 545-586.

[29] A.L. Cauchy, Cours d'analyse, (1821).

[30] A. Denjoy, Mémoire sur les nombres dérivés des fonctions continues, J. Math. Pure Appl., 7 no. 1 (1915), 123-125.

[31] A. Denjoy, Sur les fonctions dérivées sommables, Bul. Soc. Math. France, 43 (1915), 161-248.

[32] A. Denjoy, Sur les courbes definie par les equations différentielles à la surface du tore, J. Math Pure et Appl., 11 no. 29 (1932), 333-375.

[33] R. L. Devaney, An introduction to chaotic systems, (1989) Addisonwestley Publishing Co. Inc., Redwood City, California.

[34] P. Du Bois-Reymond, Die Allgemeine Functionentheorie I, Tübingen (1882).

[35] M. J. Evens, P. D. Humke, C. Lee, R. J. O'Malley, Corrigendum to characterizations of turbulent one-dimensional mappings via $\omega$-limit sets, Trans. Amer. Math. Soc., 333 no.2 (1992) 939-940.

[36] M. J. Evens, P. D. Humke, C. Lee, R. J. O'Malley, Characterizations of turbulent one-dimensional mappings via $\omega$-limit sets, Trans. Amer. Math. Soc., 326 no.1 (1991) 261-280.

[37] P. Fatou, Sur les equations fonctionnelles, Bull. Soc. Math. France, 47 (1919), 161-271.

[38] P. Fatou, Sur les equations fonctionnelles, Bull. Soc. Math. France, 48 (1920), 33-94;208-314.

[39] Fréchet, Thesis, Rend. del Circolo Matem di Palermo 22 (1906), 1-100.

[40] Liao Gongfu, $\omega$-limit sets and chaos for maps of the interval, Northeastern Math. J. 6 no. 2 (1990) 127-135

[41] R. Graw, On the connection between periodicity and chaos of continuous real functions and their iterates, Aeq. Math. 19 (1979) 277-278

[42] J. Guckenheimer, Sensitive dependence on initial conditions for onedimensional maps, Comm. Math. Phys., 70 (1979), 133-160. 
[43] M Handel, A pathological area preserving $C^{\infty}$ diffeomorphism of the plane, Proc. Amer. Math. Soc. 86 (1982), 163-168.

[44] T. Hedberg, The Kolmogorov superposition theorem: Appendix II, Lecture Notes in Mathematics: Topics in Approximation Theory 187 (1971), Springer-Verlag, New York 267-275.

[45] D. Hilbert, Mathematische Probleme, Bull. Amer. Math. Soc. Ser. 2, 10 no. 8 (1902), 437-479.

[46] C. E. Hobson, Theory of functions of a real variable, 1957 Dover, New York.

[47] J. G. Hocking and G.S. Young, Topology, 1961, Addison-Wesley, Reading Massachusetts.

[48] F. Hofbauer and G Keller, Some remarks about the recent results about S-unimodal maps, Ann. Inst. Henri Poincaré, 53 no. 4 (1990), 413-425.

[49] P. D. Humke and M. Laczkovich, Approximations of continuous functions by squares, Ergod. Th. and Dynam. Sys., 10 (1990), 361-366.

[50] P. D. Humke and M. Laczkovich, The Borel structure of iterates of continuous functions, Proc. Edinburgh Math. Soc., 32 (1989) 483-494.

[51] P. D. Humke and M. Laczkovich, Typical continuous functions are virtually nonmonotone, Proc. Amer. Math. Soc., 94 no.2 (1985) 244-248.

[52] S. H. Jones, The Baire Category Theorem: It's Scope (Thesis), University of California, Santa Barbara (1995).

[53] G. Julia, Mémoire sur l'iteration des fonctions rationales, J. Math., 8 (1918), 47-245.

[54] Judy A. Kennedy and James A. Yorke, Bizarre topology is natural in dynamical systems, Bull. Amer. Math. Soc., 32 no. 3 (1995), 309-316.

[55] Bernd Kirchheim, Descriptive mapping properties of typical continuous functions, Real Anal. Exch., 20 no.1 (1994/95), 359-362.

[56] B. Knaster, An indecomposible plane continuum which is homeomorphic to each of its nondegenerate subcontinua, Fund. Math., 3 (1922), 247.

[57] B. Knaster, Sur les ensembles connexes irréductibles, Fund. Math., 10 (1927), 277. 
[58] C. Kuratowski, Topologie I, and II 4th ed., Monographie Matematyczne 20 . Warszawa (1958).

[59] M. Kuchta and J. Smital, Two points scrambled set implies chaos, Proc. Europ. Conference on Iteration Theory, Caldas de Malavella, Spane 1987, World Scientific, Singapore (1989), 427-430.

[60] Herve Lehning, Dynamics of typical continuous functions, Preprint Proc. Amer. Math. Soc., (1993).

[61] T.-Y. Li and J.A. Yorke, Period three implies Chaos, Amer. Math. Monthly, 82 (1975), 985-992.

[62] G. G. Lorenz, Proceedings and Symposia in Pure Mathematics 28 (1976), 419-430.

[63] R. M. May, Simple mathematical models with very complicated dynamics, Nature, 261 (1976), 459-467.

[64] S. Mazurkiewicz, Sur les fonctions non-dérivables, Studia Math., 3 (1932), 92-94.

[65] S. Mazurkiewicz, Fund. Math., 2 (1921), 286.

[66] S. Mazurkiewicz, Fund. Math., 10 (1927), 305.

[67] W. de Melo, and S. van Strien, A structure theorem in one-dimensional dynamics, Annels of Math., 129 (1989), 519-546.

[68] J. Milnor, On the concept of attractor, Commun. Math. Phys., 99 (1985), $177-195$.

[69] M. Misiurewicz, Horseshoes for mappings of the interval (Russian), Bull. Acad. Pol. Sci., 27 (1979), 167-169.

[70] M. Misiurewicz, Absolutely continuous measures of certain maps of the interval, Publ. Math. IHSE, 53 (1981), 17-51.

[71] E. E. Moise, An indecomposible plane continuum which is homeomorphic to each of its nondegenerate subcontinua, Trans. Amer. Math. Soc., 63 (1948), 583 .

[72] E. E. Moise, A note on the pseudo-arc, Trans. Amer. Math. Soc., 64 (1948), 57-58.

[73] A. P. Morgenstern, Unendlich oft differenzierbare nicht-analytische funktionen, Mathematiche Nachrichten, 44 (1938), 74. 
[74] A. Ostrovski, Uber Dirichletsche reinen und algebraische differentialgleichungen, Math. Z. 8 (1920), 241-298.

[75] J. C. Oxtoby, Measure and Catagory, Springer-Verlag, New York (1971).

[76] J. C. Oxtoby, A note on transitive transformations, Proc. Nat. Acad. Sci. U.S., 23 (1937).

[77] C. Preston, Iterates of maps of the interval, Lecture Notes in Mathematics, 999, Springer (1983).

[78] D. Preiss and J. Tišer, Points of nondifferentiability of typical Lipschitz functions, Real Anal. Exchange, 20 no. 1 (1994-95), 219-226.

[79] A. N. Šarkovskii, Attracting sets containing no cycles (Russian), Ukrain. Math. J., 20 (1968), 136-142.

[80] A. N. Šarkovskii, The partially ordered system of attracting sets,Dolk. Akad. Nauk. SSSR 170 (1966), no. 6, 1384-1386.

[81] A. Schwartz, A gereralization of the Poincaré-Bendixison theorem to closed two dimensional maniforlds, Amer. J. Math., 85 (1963), 453-458.

[82] K. Simon, On the periodic points of typical continuous functions, Proc. Amer. Math. Soc., 105 no. 1 (1989), 244-249.

[83] D. Singer, Stable orbits and bifrucations of maps of the interval, SIAM J. Appl. Math., 35 (1978), 260-267.

[84] J. Smítal, Chaotic functions with zero topological entropy, Trans. Amer. Math. Soc., 297 no. 1 (1986), 269-282.

[85] D. A. Sprecher, A survey of solved and unsolved problems on superposition of functions, J. of Approximation theory, 6 no. 2 (1972), 417-431.

[86] D. A. Sprecher, On the functional complexity of the superposition of functions, Real Anal. Exch., 9 no.2 (1983/84), 417-431.

[87] J. Sternfeld, Dimension, superposition of functions and separation of points in compact metric spaces, Math. Publication Series, Univ. of Haifa, Report 74 (1983).

[88] Albert Torchinski, Real Variables, 1988, Addison wesley, Redwood City.

[89] Vitushkin, On Hilbert's 13th problem, Dolk. Akad. Nauk SSSR, 95 (1954), 701-704. 
[90] C.E. Weil, On nowhere monotonic functions, Proc. Amer. Math. Soc., 56 (1976), 388-389.

[91] Wójtowicz, The typical structure of the sets $\{x: f(x),=h(x)$,$\} for f$ continuous and $h$ lipschitz, Trans. Amer. Math. Soc., 289 (1985), 471484 .

[92] K. Yoneyama, Theory of continuous sets of points, Tohoku Math. J, 1112 (1917), 43-158 